suministrada se caracterizan por su inadaptación a-las condiciones del medio, del clima y por el desconocimiento que en su elaboración se ha hecho, de los recursos materiales propios de las regiones.

4. La supervisión escolar, tanto a nivel de la administración de la Iglesia como a nivel de la administración del Estado es deficiente. En la Iglesia, esta función es desempeñada en su mayor parte por personas ajenas a la labor docente.

La supervisión, por parte del Estado, se ve obstaculizada por los altos costos de transporte, escaso número de supervisores, permanente ubicación de éstos en las sedes centrales, y su apatía por el desempeño de esta funciones en las regiones más apartadas.

5. El número de alumnos matriculados en los territorios bajo contrato pertenecientes a los dos sectores, ascendía en 1977 a 950.990 estudiantes distribuidos conforme el siguiente cuadro:

\begin{tabular}{|l|c|c|c|}
\hline \multicolumn{1}{|c|}{ Nivel } & PRIMARIA & MEDIA & TOTAL \\
\hline Administrac. & & & \\
\hline IGLESIA & 50.707 & 15.210 & 65.917 \\
\hline ESTADO & 772.361 & 112.712 & 885.073 \\
\hline TOTAL & 823.068 & 127.922 & 950.990 \\
\hline
\end{tabular}

6. La modalidad de educación media que se ofrece con mayor frecuencia es el bachillerato académico, que no responde a las necesidades de capacitación laboral específicas para el área rural, lo que ha ocasionado que los alumnos centren sus aspiraciones en el logro de una vinculación con la docencia o con la administración pública de los municipios.

\title{
EVALUACION DE LAS ESCUELAS NORMALES DE COLOMBIA
}

\section{Antecedentes}

Uno de los principales mecanismos para lograr el mejoramiento cualitativo de la educación lo constituye, por un lado, la capacitación y perfeccionamiento del personal docente en servicio y, por otro, la elevación de los niveles cualitativos de formación de los futuros maestros. Es obvio que aún el mejor de los programas de capacitación perdería a corto plazo su eficacia o impacto silos egresados normalistas de cada año, que van reemplazando a los maestros en servicio, no tuvieran la preparación adecuada para elevar la calidad de la enseñanza que se está impartiendo en las escuelas primarias del país. De ahí que el conocimiento objetivo del funcionamiento y la calidad académica de las escuelas normales, con miras a tomar las medidas que puedan conducir al mejoramiento cualitativo de la educación básica primaria, es de una necesidad evidente.

Por otro lado, la situación de la oferta y demanda de profesores normalistas ofrece algunos indicadores concretos que vislumbran el problema de incorporación al sistema de educación, por saturación de la demanda o por acción de otros factores. Para una apreciación más profunda de la situación es necesario hacer estimaciones más precisas tomando en cuenta el stock acumulado de maestros egresados en períodos anteriores, la sustitución de maestros por personas con otras calificaciones, la deserción profesional, etc. 
En documentos elaborados anteriormente ${ }^{42}$ se señalan diversos problemas estructurales de la modalidad normalista, en particular aquellos referidos a la concentración excesiva de planteles en una misma área geográfica y a las situaciones de superávit y déficits de egresados en relación con el número de maestros necesarios' para atender la matrícula en el nivel de primaria. Si bien estos documentos muestran realidades referidas a la época en que fueron elaborados, se planteaba la necesidad de analizar la situación actual de la educación normalista en el país.

\section{Objetivos generales}

El estudio tuvo como finalidad establecer los principales problemas que se presentan en la enseñanza normalista, a fin de proporcionar criterios al Ministerio de Educación que puedan conducir a un mejor funcionamiento y a una mejor calidad de esta modalidad, y adecuar el número de establecimientos y de egresados a las condiciones y necesidades reales del país.

Los objetivos generales fueron los siguientes:

- Evaluar los insumos, el funcionamiento y calidad académica de las escuelas normales del país.

— Evaluar la administración y supervisión del sistema normalista.

- Efectuar estimaciones de la situación de oferta y demanda de profesores para las escuelas primarias, a nivel nacional.

\section{Objetivos específicos}

1. Determinar el número, ubicación y clasificación de los establecimientos que imparten enseñanza normalista en el país.

2. Establecer los recursos físicos y presupuestarios de la modalidad.

3. Analizar el marco legal y las condiciones reales de funcionamiento en cuanto a sus aspectos administrativos y docentes.

4. Determinar la calidad académica de los profesores de las Escuelas Normales y de sus respectivas escuelas anexas.

5. Buscar indicadores objetivos, a nivel de los estudiantes, de la calidad de la enseñanza impartida por las escuelas normales.

6. Establecer las formas y el contenido de la administración a nivel central.

7. Analizar la efectividad y el cumplimiento de las acciones de evaluación en esta área.

\footnotetext{
${ }^{42}$ M.E.N. "proyecto de clasificación y reubicación de las Normales existentes de acuerdo con el Decreto No. 088 de 1976". Bogotá, 1976.

Departamento Nacional de Planeación "proyecto para el desarrollo de un programa integrado de formación y capacitación docente para el sistema educativo colombiano". Bogotá, 1972.
} 
8. Estimar el número de egresados de las Escuelas Normales del país en el período 1957-1977.

9. Establecer el número de profesores en ejercicio y hacer estimaciones de la demanda en corto plazo.

10. Comparar la oferta y la demanda de maestros.

\section{Metodología}

En la evaluación se empleó un diseño comparativo, utilizando variables y normas de contrastación con otras modalidades de la educación media, a través de un enfoque descriptivo.

Las principales fuentes de información fueron el personal directivo, docente, administrativo y de alumnos, quienes diligenciaron cuestionarios elaborados con base en las variables seleccionadas.

Otras fuentes fueron las Secretarías de Educación, los Delegados de los Fondos Educativos Regionales y el Instituto Colombiano para el Fomento de la Educación Superior.

El universo del estudio estuvo conformado por los 209 planteles de enseñanza normalista que funcionaban en el país en 1977 y los que se terminaron en el período 1970-1976, equivalentes a un total de 231 establecimientos de la modalidad.

A fin de lograr un mejor conocimiento de la calidad del personal directivo y docente, se tomó una muestra de cincuenta planteles con sus respectivas escuelas anexas, oficiales y no oficiales. El número de profesores en esa muestra ascendió a 1.750 .

Para la realización de la evaluación institucional se seleccionó una muestra de 18 planteles, con el fin de obtener información directa de la organización, funcionamiento y administración a través del personal directivo, docente, administrativo, alumnos y algunos miembros de la comunidad.

La clasificación global de las normales según calidad académica y recursos didácticos se hizo mediante la técnica de análisis de conglomerados y análisis discriminante, con la utilización de un programa de computador.

\section{Resultados}

La investigación dio lugar a un extenso número de conclusiones y recomendaciones tales como la existencia de un excesivo número de planteles que ha ocasionado una sobre-saturación de maestros. Se calcula una cifra aproximada de 28.000 docentes en espera a ser incorporados al sistema educativo, cifra que sumada al número de maestros en ejercicio, alcanzaría a cubrir la demanda de los próximos tres o cuatro años sin necesidad de recurrir a nuevas promociones, aun elevando la tasa de escolaridad al $85 \%$ con una mejor utilización de los recursos existentes.

Las permanentes reformas a los programas de formación normalista no tienen como base previas evaluaciones. Tales reformas han carecido de un proceso técnico, de pre- 
visión real del mejoramiento cualitativo y del número de maestros requeridos para la educación primaria a nivel nacional.

En la organización actual de la escuela normal no se aprecia interés por dirigir el esfuerzo educativo hacia la formación del futuro maestro dentro de la realidad del medio en donde deba desenvolverse, para que pueda tener un conocimiento real de los problemas, que le permita analizarlos y participar activamente en sus soluciones; estos factores han sido la causa del distanciamiento, de la limitada participación y apoyo de la comunidad en la tarea educativa.

La deficiente calidad de las Escuelas Normales se refleja claramente en la clasificación que se hizo de ellas a nivel nacional, en la que se observa que más del $50 \%$ no cumplen los requisitos que garanticen la formación de maestros idóneos. Esta afirmación se demuestra con los resultados de las pruebas del ICFES en las cuales uno de los promedios más bajos fue el obtenido por los egresados de las normales, acusando mayor deficiencia los de las instituciones no oficiales.

Ante estos resultados el estudio propone la conversión del bachillerato pedagógico a carrera intermedia ofreciendo dentro de ésta, áreas de especialización.

Además plantea la necesidad de reducir a 60 el número de instituciones de formación normalista, adoptando las restantes, otras modalidades más acordes con las necesidades del país y, establecer una unidad técnica en el Ministerio de Educación Nacional que canalice las políticas de la educación normalista, asesore las Escuelas Normales y se constituya en el centro de comunicación y coordinación con las diferentes dependencias del nivel central y de las entidades regionales.

\section{EVALUACION DE LA EDUCACION BASICA PARA ADULTOS}

\section{Planeamiento del problema}

La educación de adultos en el país inició su proceso de configuración formal a partir de 1936, con la creación del Servicio Escolar Voluntario. En los años posteriores ha sido objeto de mayor atención por parte del Estado, lo que le ha permitido desarrollarse en una magnitud considerable dentro del conjunto educacional colombiano, sin que alcance aún respuestas adecuadas a la demanda educativa de la población adulta.

Los esfuerzos que se vienen realizando a nivel nacional en el campo de la educación de adultos por parte del Estado, están concentrados principalmente en el Ministerio de Educación Nacional y en el Fondo de Capacitación Popular de la Presidencia de la República.

En el sector privado los programas de ACPO tienen esta misma función. Se encuentran, además, otras entidades con cubrimiento regional como el Centro Laubach de educación básica de adultos, en Antioquia.

A pesar de los avances logrados por medio de la enseñanza directa, y programas educativos de radio y televisión, la demanda por este tipo de educación aumenta en la 